\title{
REDUCCIÓN DE COSTO DE FABRICACIÓN DE ACEROS PARA LA CONSTRUCCIÓN MEDIANTE AMPLIACIÓN DE LOS LÍMITES DE ESPECIFICACIÓN*
}

Valeria Romina Stricker ${ }^{1}$ Paola Carolina Torrico ${ }^{2}$ Francisco Casarini

\section{Resumen}

Acerbrag es una empresa Argentina siderúrgica de 50 años de trayectoria, desde el año 2007 pertenece al grupo Votorantim de origen Brasilero. Se dedica a la elaboración de aceros largos para la construcción. La planta industrial cuenta con un proceso de acería integrado, horno eléctrico, horno cuchara y máquina de colada continua. La materia prima es $100 \%$ chatarra, característica que la diferencia del resto de las acerías del país. Debido a esto el enfoque principal de este proyecto es reducir el costo de fabricación mediante un "empobrecimiento" de la carga metálica a partir de la flexibilización de los límites de especificación. Esto implica un mayor control del desempeño de los procesos a fin de detectar posibles desvíos, para ello fue necesario implementar la utilización de herramientas estadísticas y gráficas para su monitoreo. La modificación en el mix de fabricación no muestra una alteración significativa en la composición química y propiedades mecánicas de los productos terminados, situación favorable para la continuidad del proyecto. Se observa además, de la reducción de costo esperada, una ventaja en la operación del patio de chatarra y el proceso de acería y en la utilización de chatarras con altos niveles de residuales tales como $\mathrm{Cr}-\mathrm{Ni}$.

Palabras-clave: Carga metálica; "Empobrecimiento”; Mix; Familia de chatarra.

\section{REDUCED COST OF MANUFACTURING STEEL FOR THE CONSTRUCTION BY INCREASING THE SPECIFICATION LIMITS}

\section{Abstract}

Acerbrag is an Argentine steel company over 50 years of experience that since 2007 belongs to the steel unit of the Votorantim group of Brazilian origin. It is dedicated to the production of long steel for construction. The industrial plant has an integrated steelmaking process, electric furnace, ladle furnace and continuous casting machine. The raw material of this mill is $100 \%$ scrap, different characteristic from other mills in the country. Given that the raw material is $100 \%$ scrap the primary focus of this project is to reduce the manufacturing cost through a "impoverishment" of the metal loading from flexible specification limits. Increase the specification limits for closer monitoring of the performance of processes to detect possible deviations that lead to refuse material breaches of technical specification, for it was necessary to implement the use of statistical and graphical tools for monitoring. Changes in the mix of production did not show significant changes in mechanical properties and chemical compositions of products, favorable situation for the continuation of this project. It is observed besides the expected cost reduction, an advantage in operating the scrap yard and mill process as well as the possibility of using scrap with high levels of waste such as $\mathrm{Cr}-\mathrm{Ni}$.

Keywords: Metal load; "Impoverishment"; Mix; Family scrap.

Ingeniera Metalúrgica, Jefe, Calidad, Acerbrag, Bragado, Buenos Aires, Argentina.

Ingeniera Metalúrgica, Ingeniera, Calidad, Acerbrag, Bragado, Buenos Aires, Argentina.

Técnico, Analista, Calidad, Acerbrag, Bragado, Buenos Aires, Argentina. 


\section{INTRODUCCIÓN}

El principal objetivo del proyecto de modificación de especificación está orientado a:

1. Reducir el costo del mix de chatarra

2. Aumentar la utilización de chatarra de menor costo (viruta y hojalata)

3. Mejorar la rotación de los tipos de chatarra en stock

4. Flexibilizar el mix de carga de 5 tipos a 7 incluyendo chatarra con altos residuales.

5. Ventaja en la negociación de compra de chatarra.

El alcance del mismo se corresponde al $80 \%$ de la producción total de Acerbrag, siendo potencial su aplicación en un 15\% más correspondiente a la calidad 1010.

Actualmente las modificaciones fueron implementadas en las calidades 1020 y 1040.

\section{DESARROLLO}

\subsection{Análisis Estadístico - Propiedades Mecánicas}

Las calidades 1040 y 1020 se utilizan para la fabricación de barras de acero conformadas para armaduras en estructuras de hormigón. Este producto debe cumplir con los requerimientos indicados en las Normas de aplicación obligatoria IRAM-IAS U500-528 e IRAM-IAS U500-206 está última de característica soldable.

Para obtener los nuevos límites de especificación, se realizó análisis estadístico de previsibilidad de propiedades mecánicas en las barras ADN y ADN-S, utilizándose para el estudio, una base de resultados de seis meses de ensayos mecánicos de estos productos.

Las ecuaciones de regresión obtenidas fueron las siguientes:

Tabla 1. Ecuaciones de regresión SAE 1040

\begin{tabular}{|l|l|l|}
\hline $\begin{array}{l}\text { Propiedad } \\
\text { mecánica }\end{array}$ & Ecuación de regresión & $\begin{array}{l}\text { Error medio (valores } \\
\text { reales vs ecuación) }\end{array}$ \\
\hline Tracción (Mpa) & $\begin{array}{l}\text { Trac }=538+336 \mathrm{C}+95,8 \mathrm{Mn}-176 \mathrm{Si}-918 \mathrm{~S}+21,7 \mathrm{Cu}+ \\
272 \mathrm{Sn}+20,6 \mathrm{Ni}+16,2 \mathrm{Cr}-1237 \mathrm{~V}\end{array}$ & $2,4 \%$ \\
\hline Fluencia (Mpa) & $\begin{array}{l}\text { Flue. }=356+225 \mathrm{C}+19,7 \mathrm{Mn}-29,6 \mathrm{Si}+55,9 \mathrm{~S}+6,1 \mathrm{Cu}- \\
104 \mathrm{Sn}-32,4 \mathrm{Ni}+55,4 \mathrm{Cr}-233 \mathrm{Mo}-1143 \mathrm{~V}\end{array}$ & $2 \%$ \\
\hline $\begin{array}{l}\text { \% } \\
\text { Alargamiento }\end{array}$ & $\begin{array}{l}\mathrm{Al} \%=29,5-11,7 \mathrm{C}-3,68 \mathrm{Mn}-7,69 \mathrm{Si}+36,6 \mathrm{P}-28,6 \mathrm{~S}- \\
7,00 \mathrm{Cu}-29,9 \mathrm{Sn}+11,9 \mathrm{Ni}-1,15 \mathrm{Cr}+268 \mathrm{Al}-286 \mathrm{~V}\end{array}$ & $8,4 \%$ \\
\hline
\end{tabular}

Tabla 2. Ecuaciones de regresión SAE 1020

\begin{tabular}{|l|l|l|}
\hline $\begin{array}{l}\text { Propiedad } \\
\text { mecánica }\end{array}$ & Ecuación de regresión & $\begin{array}{l}\text { Error medio (valores } \\
\text { reales vs ecuación) }\end{array}$ \\
\hline Tracción (Mpa) & $\begin{array}{l}\text { Trac. }=508+464 \mathrm{C}-62,2 \mathrm{Mn}+106 \mathrm{Si}+1745 \mathrm{P}+24,9 \mathrm{Cu}- \\
104 \mathrm{Sn}+266 \mathrm{Ni}-255 \mathrm{Mo}+955 \mathrm{Al}-4527 \mathrm{~V}\end{array}$ & $5,4 \%$ \\
\hline Fluencia (Mpa) & $\begin{array}{l}\text { Flue. }=518+124 \mathrm{C}-113 \mathrm{Mn}+128 \mathrm{Si}+1993 \mathrm{P}-33,8 \mathrm{Cu}- \\
582 \mathrm{Sn}+248 \mathrm{Ni}+65,7 \mathrm{Cr}-245 \mathrm{Mo}-1121 \mathrm{~V}\end{array}$ & $8,3 \%$ \\
\hline $\begin{array}{l}\text { \% } \\
\text { Alargamiento }\end{array}$ & $\begin{array}{l}\mathrm{Al} \%=13,8-3,50 \mathrm{C}+6,67 \mathrm{Mn}-2,36 \mathrm{Si}-48,8 \mathrm{P}+2,86 \mathrm{Cu}+ \\
19,2 \mathrm{Sn}-14,6 \mathrm{Ni}-9,10 \mathrm{Cr}+33,0 \mathrm{Mo}-28,0 \mathrm{~V}\end{array}$ & $11,8 \%$ \\
\hline
\end{tabular}

Se observa un error medio mayor en la calidad 1020, esto se debe a que las propiedades mecánicas son obtenidas a partir de tratamiento térmico en línea (QTB) y depende de la profundidad del temple, mientras que para el SAE 1040, las propiedades mecánicas son resultado de la composición química. 


\subsection{Pruebas Experimentales - Composición Química}

En una primera etapa, las modificaciones en la composición química, estuvieron orientadas a aumentar los niveles aceptables de los elementos Azufre, Fósforo y los residuales $\mathrm{Cr}, \mathrm{Ni}, \mathrm{Cu}, \mathrm{Sn}$ y $\mathrm{Mo}$.

Analizado el comportamiento de esta nueva especificación, durante tres meses, se planifica tener una segunda etapa de modificación basada únicamente en el incremento de los niveles de $\mathrm{Cr}$ en ambas calidades.

En la Tabla 3, se observa un comparativo de la suma total de estos elementos antes y después de la primera modificación.

Tabla 3. Aumento de residuales, $\mathrm{P}$ y $\mathrm{S}$

\begin{tabular}{|l|l|l|l|l|}
\hline Calidad & $\begin{array}{l}\text { Suma } \\
\text { residuales }\end{array}$ & Aumento (\%) & Suma P + S & Aumento (\%) \\
\hline 1040 & 0,830 & $28 \%$ & 0,070 & $14 \%$ \\
\cline { 1 - 2 } $1040-$ MOD & 1,060 & & 0,080 & \\
\hline 1020 & 0,702 & $47 \%$ & 0,065 & 31 \\
\cline { 1 - 1 } $1020-$ MOD & 1,060 & & 0,085 & \\
\hline
\end{tabular}

Se tiene un incremento total entre ambas calidades de $75 \%$ en los niveles de residuales y $45 \%$ en la suma de $P$ y $S$.

Esta suba permite modificar el mix de fabricación anulando el uso de chatarra de bajos residuales, chatarra de mayor costo, en el mix actual e introducir en el nuevo mix modificado viruta y hojalata, chatarra de menor costo.

\subsection{Metodología de los Cambios - Mix de fabricación}

Para poder observar el real impacto de la composición química en los productos terminados se desarrolla en adelante la modificación correspondiente a la calidad SAE 1040 utilizada en barras ADN.

Durante el periodo de prueba se definió un primer mix cuyo objetivo fue obtener un análisis químico lo más próximo a los límites máximos de especificación, para validar el comportamiento mecánico del producto terminado.

En la Tabla 4 se indican los porcentajes de cada mix por familia de chatarra. Cuando se habla de "familia" de chatarra corresponde a un grupo de dos o más clases de chatarra de similares características.

Los principales cambios que se observan son:

- Eliminación de chatarra de bajos residuales

- Disminución considerable en el porcentaje de chatarra tipo mezcla

- Incorporación de viruta, hojalata y fundición

Tabla 4. Mix de carga

\begin{tabular}{|l|l|l|}
\hline Tipo de chatarra & Mix Actual 1040 & Mix Prueba \\
\hline Mezcla & $25 \%$ & $16 \%$ \\
\hline Pesada & $45 \%$ & $44 \%$ \\
\hline Paquete viejo & $0 \%$ & $11 \%$ \\
\hline Retorno & $8 \%$ & $7 \%$ \\
\hline Bajo Residuales & $22 \%$ & $0 \%$ \\
\hline Viruta & & $8 \%$ \\
\hline Hojalata & & $7 \%$ \\
\hline Fundición & & $7 \%$ \\
\hline
\end{tabular}


Con este mix se llegaron a los resultados indicados en las Figuras 1 y 2.

En la Figura 1 se comparan las coladas de mix de prueba con coladas de mix actual. En el mix de prueba se tiene un mayor incremento en el \% $\mathrm{Sn}$ y en el \%Cu, \%Cr y $\% \mathrm{Ni}$ este incremento es menos acentuado. En esta prueba, se mantuvieron valores de \%Mn algo menores para no alterar significativamente el Ceq.

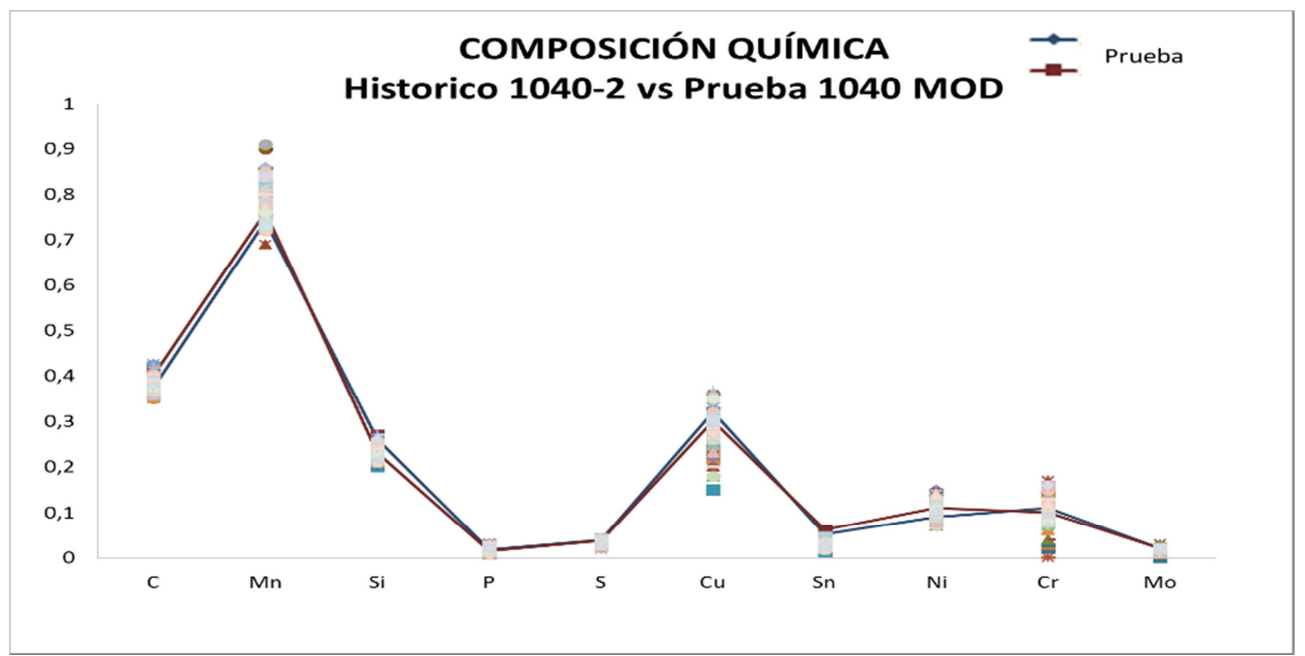

Figura 1. Comparativo composición química prueba vs histórico

En la Figura 2 se comparan las propiedades mecánicas de las coladas de mix de prueba con las de mix actual en barras ADN diámetro $8 \mathrm{~mm}$.

Se observa un buen comportamiento de los valores de tracción, fluencia y alargamiento respecto de los valores mínimos exigibles en la Norma IRAM-IAS U500-528.

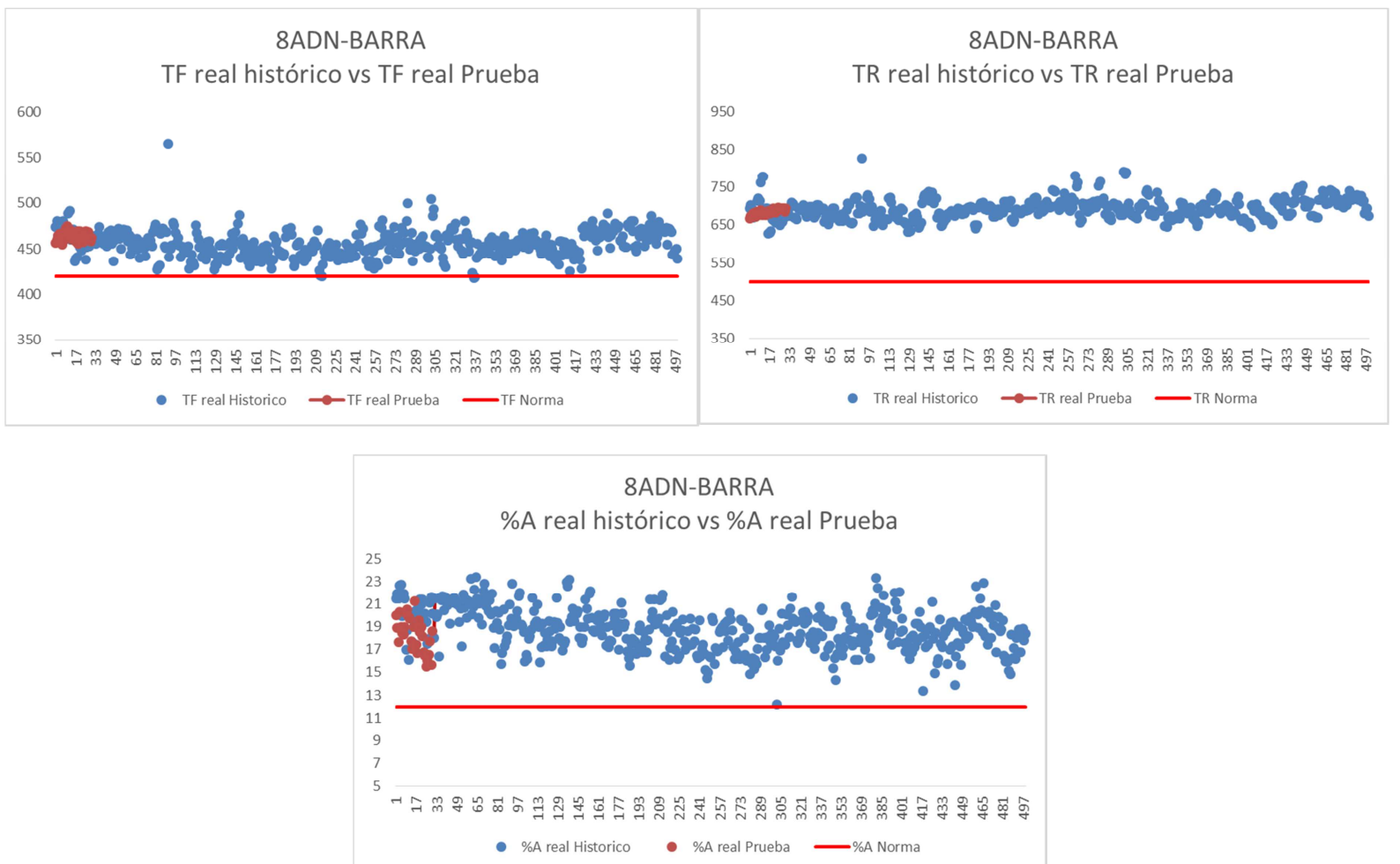

Figura 2. Comparativo ensayos mecánicos prueba vs mínimo de Norma 


\section{RESULTADOS Y DISCUSIÓN}

\subsection{Análisis de Costo Mix Modificado}

Realizadas todas las pruebas se procedió a la modificación del mix teniendo en cuenta el costo de cada familia de chatarra. El mix definido se muestra en la Tabla 5.

Tabla 5. Mix modificado

\begin{tabular}{|l|l|}
\hline Tipo de chatarra & Mix MODIFICADO \\
\hline Mezcla & $21 \%$ \\
\hline Pesada & $49 \%$ \\
\hline Paquete viejo & $11 \%$ \\
\hline Retorno & $10 \%$ \\
\hline Bajo Residuales & $0 \%$ \\
\hline Viruta & $7 \%$ \\
\hline Hojalata & $2 \%$ \\
\hline Fundición & $0 \%$ \\
\hline
\end{tabular}

Para este análisis se tuvo en cuenta el precio medio de cada familia de chatarra correspondiente al cierre de mes de enero de 2015 y los rendimientos metálicos de cada familia indicados en la Tabla 6.

Tabla 6. Rendimiento por familia de chatarra

\begin{tabular}{|l|l|}
\hline Tipo de chatarra & $\begin{array}{l}\text { Rendimiento } \\
\text { metálico }\end{array}$ \\
\hline Mezcla & $84 \%$ \\
\hline Pesada & $87 \%$ \\
\hline Paquete viejo & $82 \%$ \\
\hline Retorno & $93 \%$ \\
\hline Bajo Residuales & $92 \%$ \\
\hline Viruta & $80 \%$ \\
\hline Hojalata & $92 \%$ \\
\hline Fundición & $90 \%$ \\
\hline
\end{tabular}

\subsection{Ganancias Esperadas}

Con el mix modificado se llega a una reducción en el costo de fabricación de 3,5\% por tonelada de palanquilla producida. Esta reducción alcanza al $80 \%$ de la producción total de acería.

La ganancia esperada de este proyecto está relacionada con el cumplimiento del mix modificado.

En la figura 3 se muestra el porcentaje de ganancia real vs esperada. Se observa que la implementación de este cambio tuvo mejores resultados en el mes de enero alcanzando el $46 \%$ de la ganancia esperada. 
Porcentaje de Ganacia Real vs Esperada

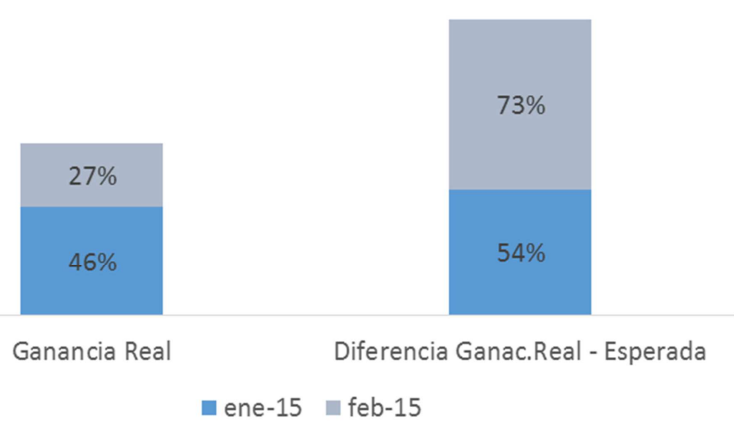

Figura 3. Ganancia esperada vs real.

El bajo cumplimiento estuvo afectado por los siguientes factores:

- Menor consumo de chatarra pesada, por bajo nivel de volumen de compra.

- Mayor consumo de mezcla, como principal reemplazo de pesada.

- Menor consumo de retorno interno, por falta de material procesado.

- Mayor consumo de bajos residuales para diluir el pie líquido, toda vez que el análisis químico de los residuales dio un resultado próximo a 0,15.

- Menor consumo de viruta por condiciones operativas (falta de personal, equipamiento fuera de servicio por mantenimiento)

\subsection{Monitoreo del Mix Modificado}

Para monitoreo de cumplimiento de la carga del mix se realizó una base informática en el programa Qlikview donde se observa:

- Mix teórico modificado vs real

- Costo carga metálica teórica vs real

- Composición química especificada vs real.

La figura 4 indica el cumplimiento de mix por cada familia de chatarra, cuando cada familia se corresponde al mix programado estos porcentajes deben ser del $100 \%$.

Se observa en el gráfico un cumplimiento variable entre las familias de chatarra siendo el porcentaje de cumplimiento menor en los tipos hojalata y mezcla.

El no cumplimiento en el porcentaje de viruta tiene un impacto negativo en el costo total del mix y en la pérdida de su rendimiento metálico por degradación debido al alto tiempo en stock.

Los mejores resultados se observan en las familias arrabio y bajos residuales.

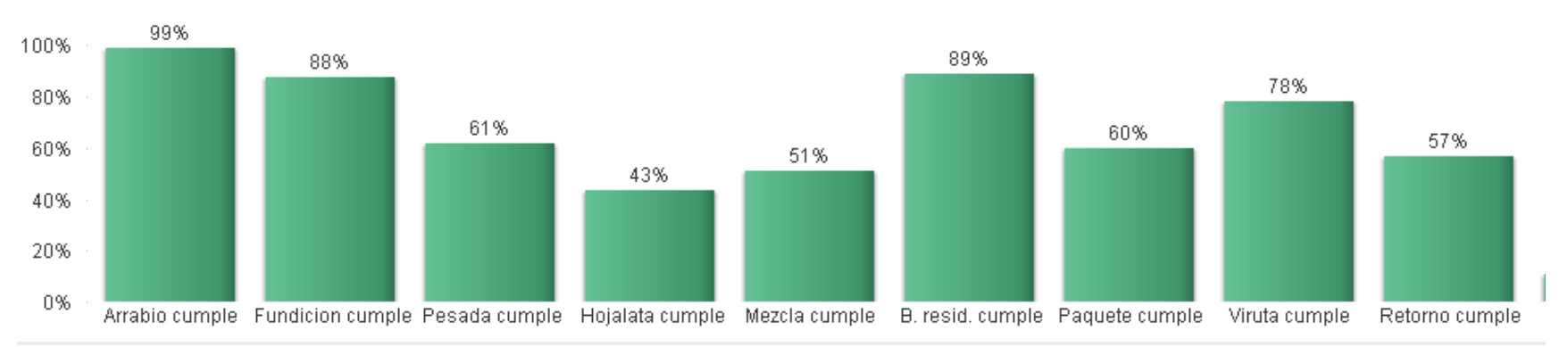

Figura 4. Cumplimiento mix de chatarra 


\section{CONCLUSIÓN}

- La modificación de los límites de especificación química de las calidades 1020 y 1040 correspondientes al $80 \%$ de la producción total de la acería de Acerbrag permitió un "empobrecimiento de la carga metálica" cuya reducción de costo en el mix de fabricación es de 3,5\%.

- La reducción de costo obtenida con este proyecto es equivalente a la fabricación de 5 coladas más por mes.

- Del análisis de estos cambios, se observa en las figuras 5 y 6 , que el perfil de composición química de las calidades modificadas se comportan dentro de los límites aceptables al igual que las propiedades mecánicas.
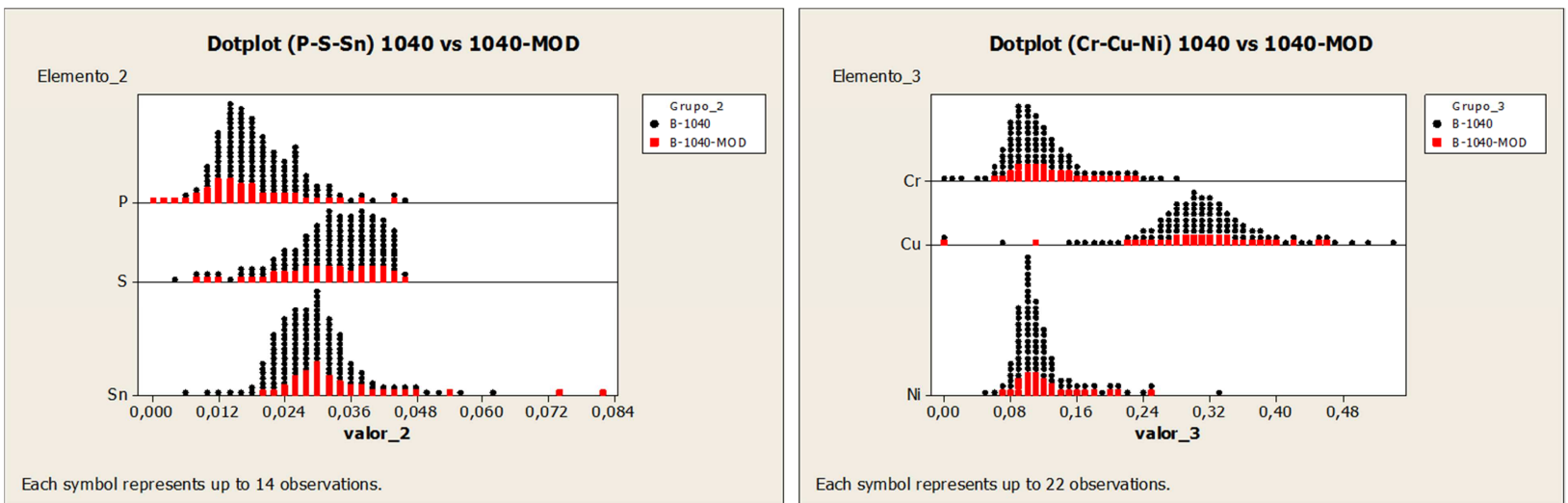

Figura 5. Composición química

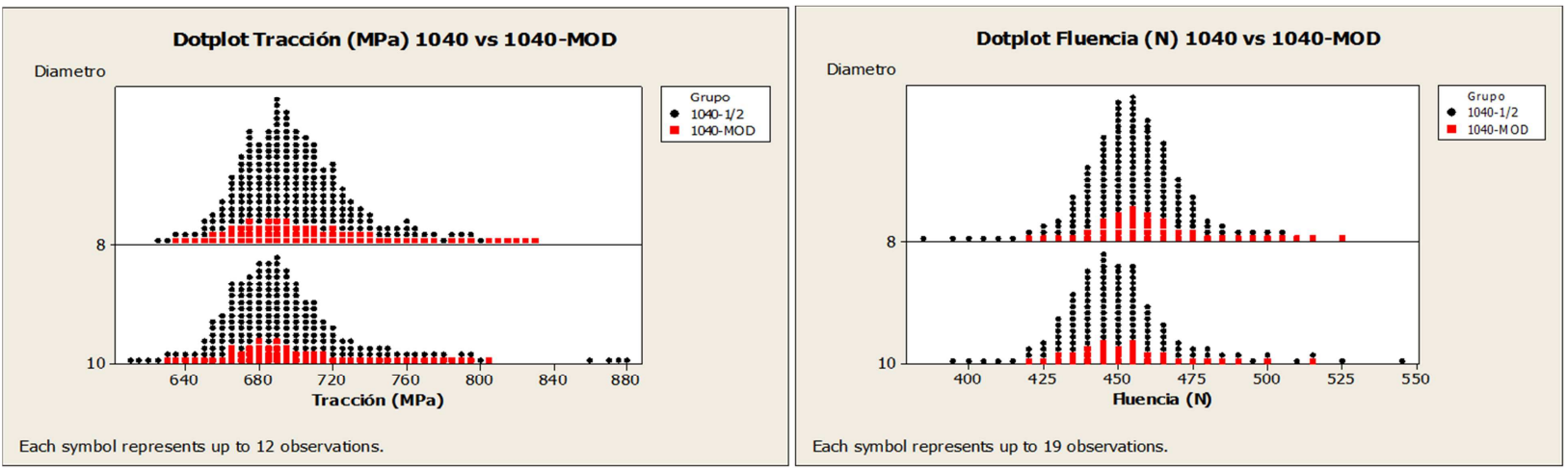

Figura 6. Propiedades mecánicas

- Ampliar los límites de especificación, lleva a la necesidad de tener controles y monitoreo de los procesos de modo más específico, teniendo que desarrollar herramientas informáticas para dicho control.

- La obtención de las ganancias esperadas, está asociada directamente con el cumplimiento de cada familia de chatarra, definida en el mix modificado, para ello es necesario desarrollar una metodología de trabajo que permita mejorar este cumplimiento. 